\title{
Inhomogeneous Cosmological Models with Flat Slices Generated from the EINSTEIN-DE SiTTER Universe
}

\author{
Hans - Jürgen Schmidt \\ Universität Potsdam, Institut für Mathematik, Am Neuen Palais 10 \\ D-14469 Potsdam, Germany, E-mail: hjschmi@rz.uni-potsdam.de
}

\begin{abstract}
A family of cosmological models is considered which in a certain synchronized system of reference possess flat slices $t=$ const. They are generated from the EINSTEIN-DE SITTER universe by a suitable transformation. Under physically reasonable presumptions these transformed models fulfil certain energy conditions.

Es wird eine Familie kosmologischer Modelle betrachtet, die in einem gewissen synchronisierten Bezugssystem flache Schichten $t=$ const. besitzen. Sie werden mittels einer geeigneten Transformation aus dem EINSTEIN-DE SITTER-Universum erzeugt. Diese transformierten Modelle erfüllen unter physikalisch sinnvollen Voraussetzungen gewisse Energiebedingungen.
\end{abstract}

\section{Introduction}

In WAINWRIGHT (1981), SCHMIDT (1982) and references cited there a class of inhomogeneous cosmological models is considered which have the 
following property: there exists a synchronized system of reference of such a kind that the slices $t=$ const. are homogeneous manifolds. Here we consider a special family of such models which possess flat slices. To this end we use the transformation formalism developed in SCHMIDT (1982). Additionally, we require that these transformations leave two coordinates unchanged; this implies the existence of a 2-dimensional Abelian group of motions. (A similar requirement is posed in WAINWRIGHT (1981), too.) Starting from a FRIEDMAN universe, we investigate whether the energy inequalities are fulfilled in the transformed model, too. In general this fails to be the case, but starting from the EINSTEIN - DE SITTER universe (EINSTEIN 1932; cf. also TOLMAN 1934) and requiring perfect fluid for the transformed model, the energy inequalities in the initial model imply their validity in the transformed model. These statements answer partially a question posed by TREDER.

\section{Models with flat slices}

The transformation formalism of SCHMIDT (1982) restricted to BIANCHI type I reads as follows: using the same notations, we have $A_{i}^{a}=\delta_{i}^{a}, \omega^{a}=d x^{a}$ and

$$
d s^{2}=-d t^{2}+g_{a b}(t) d x^{a} d x^{b}
$$

as the initial hypersurface-homogeneous model. Now let us consider the time-dependent transformation $x_{t}^{a}\left(x^{i}, t\right)$, where for each $t$ it has to be a diffeomorphism of $R^{3}$. Then one obtains

$$
g_{0 \alpha}=-\delta_{0 \alpha}, \quad g_{i j}=g_{a b}(t) x_{t, i}^{a} x_{t, j}^{b}
$$

as the transformed model. (It is no restriction to insert $g_{a b}(t)=\delta_{a b}$ in $(1)$, i.e. to start from MINKOWSKI space.)

In the following we consider only transformations which leave two coordinates unchanged, i.e. (now writing $t, x, y, z$ instead of $x^{0}, \ldots x^{3}$ resp.) 
transformations which read as follows

$$
x_{t}(x, t), \quad y_{t}=y, \quad z_{t}=z .
$$

(These we shall call $x$-transformations.) Using $x$-transformations, the KILLING vectors $\partial / \partial y$ and $\partial / \partial z$ of the initial model remain KILLING vectors. They form a 2-dimensional Abelian group of motions. All others (including the rotation $z(\partial / \partial y)-y(\partial / \partial z))$ may fail to remain KILLING vectors. On the contrary to the general case, the $x$-transformed models depend genuinely on the initial ones. In the following the 3-flat FRIEDMAN universe shall be used as initial model, i.e.

$$
g_{a b}(t)=\delta_{a b} K^{2}(t) \quad \text { with } \quad K(t)=t^{\tau} .
$$

Together with (1) one obtains

$$
\kappa \mu=\kappa T_{00}=3 \tau^{2} / t^{2}, \quad p=T_{2}^{2}=\alpha \mu \quad \text { with } \quad \alpha=\frac{2}{3 \tau}-1
$$

Now, inserting (4) in (1) and transforming to (2) with restriction (3) one obtains for the metric of the $x$-transformed model

$$
g_{11}=t^{2 \tau} \cdot\left(x_{t, 1}\right)^{2} \equiv t^{2 \tau} \cdot h(x, t), \quad g_{22}=g_{33}=t^{2 \tau}, \quad g_{\alpha \beta}=\eta_{\alpha \beta} \text { else } .
$$

This metric belongs to the so-called SZEKERES class, cf. SZEKERES (1975). Defining $a(x, t)$ by

$$
g_{11}=e^{2 a(x, t)},
$$

a coordinate transformation $\tilde{x}(x)$ yields $a(x, 1)=0$. If $v=a_{0} t$, then we have

$$
a(x, t)=\int_{1}^{t} v(x, \tilde{t}) \cdot \tilde{t}^{-1} d \tilde{t}
$$

$v(x, t)$ being an arbitrary twice continuously differentiable function which may be singular at $t=0$ and $t=\infty$. (The initial model is included by setting $x_{t}=x$, hence $a=\tau \ln t, v=\tau$.) 
For $\tau \neq 0$ different functions $v$ correspond to the same model only if they are connected by a translation into $x$-direction. Inserting (6) with (7) and (8) into the EINSTEIN equations one obtains the energy-momentum tensor

$$
\begin{array}{r}
\kappa T_{00}=\left(\tau^{2}+2 v \tau\right) / t^{2}, \\
\kappa T_{1}^{1}=\left(2 \tau-3 \tau^{2}\right) / t^{2}, \\
\kappa T_{2}^{2}=\kappa T_{3}^{3}=-v, 0 t^{-1}+\left(\tau-\tau^{2}-v \tau-v^{2}+v\right) / t^{2}, \\
\kappa T_{\alpha \beta}=0 \quad \text { else } \quad \text { and } \\
\kappa T=\kappa T_{\alpha}^{\alpha}=-2 v, 0 t^{-1}-2\left(3 \tau^{2}-2 \tau+2 v \tau+v^{2}-v\right) / t^{2} .
\end{array}
$$

The question, in which cases (6) represents a usual hypersurface-homogeneous model, can be answered as follows: metric (6) is a FRIEDMAN universe, if and only if $h_{0}=0$. For this case it is isometric to the initial model. Metric (6) is a hypersurface-homogeneous model, if and only if functions $A$ and $B$ exist for which holds $h(x, t)=A(x) \cdot B(t)$. Because of $h>0$ this is equivalent to $h_{, 01} \cdot h=h_{, 0} \cdot h_{, 1}$. In this case it is a BIANCHI type I model.

\section{$3 \quad$ Energy inequalities}

In this section it shall be discussed, in which manner the geometrically defined models described by equs. (6), ., , (9) fulfil some energy conditions. Here we impose the following conditions: each observer measures non-negative energy density, time- or light-like energy flow and space-like tensions which are not greater than the energy density. In our coordinate system these conditions are expressed by the following inequalities

$$
\begin{gathered}
T_{00} \geq\left|T_{1}^{1}\right|, \\
T_{00} \geq\left|T_{2}^{2}\right|, \\
\text { and } \quad T \leq 0 .
\end{gathered}
$$


For the initial model this means $\tau=0$ or $\tau \geq 1 / 2$, i.e. MINKOWSKI space or $-1<\alpha \leq 1 / 3$. Using the energy-momentum tensor (9), equ. (11) and (12) read

$$
\begin{gathered}
v_{, 0} t \geq-v^{2}-(3 \tau-1) v+\tau-2 \tau^{2}, \\
v_{, 0} t \leq-v^{2}+(\tau+1) v+\tau \quad \text { and } \\
v_{, 0} t \geq-v^{2}-(2 \tau-1) v+2 \tau-3 \tau^{2} .
\end{gathered}
$$

Now, if $\tau<0$, i.e. $\alpha<-1$, then (10) reads $v \leq \tau-1$; together with (14) one obtains $v_{, 0} t \leq \tau-2$. This implies the existence of a $\tilde{t}>0$ with

$v(\tilde{t}) \geq-1$ in contradiction to (10). Therefore, an initial model with $\tau<0$ (which itself contradicts the energy inequalities) cannot produce transformed models which always fulfil them.

If $\tau=0$, then (13) and (14) imply $v_{, 0} t=v-v^{2}$. This equation has the solutions $v=0$ and $v=t(t+C)^{-1}$ with arbitrary $C(x) \geq 0$. This yields $a=0$ and $a=\ln (t+C)-\ln (1+C)$ with $(7)$ and $g_{11}=1$ and $g_{11}=(t+C)^{2} \cdot(1+C)^{-2}$ resp. with (8). Then (6) shows that this is the MINKOWSKI space itself. Therefore, the MINKOWSKI space does not produce any new models.

Finally, if $\tau>0$, hence $\alpha>-1,(10)$ then reads $v \geq \max (\tau-1,1-$ $2 \tau)$. A lengthy calculation shows which transformed models fulfil the energy inequalities. For each $\tau>0$ models exist which do and models which do not fulfil them.

\section{$4 \quad$ Perfect fluid models}

The situation described above changes if one requires that the transformed model consists of perfect fluid with an equation of state. The velocity vector must be $(1,0,0,0)$ and

$$
\kappa\left(T_{1}^{1}-T_{2}^{2}\right) \equiv \frac{h_{, 0}}{(1+\alpha) t \cdot h}+\frac{1}{2 \sqrt{h}}\left(\frac{h_{, 0}}{\sqrt{h}}\right)_{, 0}=0
$$


must be fulfilled. If $f=h_{, 0} h^{-1 / 2}$, then (16) reads

$$
\frac{f}{(1+\alpha) t}+\frac{f_{, 0}}{2}=0
$$

hence $f_{, 0} f^{-1}$ does not depend on $x$, therefore $f_{, 01} \cdot f=f_{, 0} \cdot f_{, 1}$, and we can use the ansatz $f=a(t) \cdot b(x)$. Inserting this into (16), one obtains

$$
h=\left[b(x) \cdot t^{(\alpha-1)(\alpha+1)}+c(x)\right]^{2}, \quad \text { where } \quad c(x)=\sqrt{h(x, 0)}
$$

with arbitrary non-negative functions $b$ and $c$ fulfilling $b(x)+c(x)>0$ for all $x$. For energy density and pressure we then obtain

$$
\begin{array}{r}
\kappa \mu=\frac{4}{3(1+\alpha)^{2} t^{2}}-\frac{4(1-\alpha) \cdot b}{3(1+\alpha)^{2} t\left(b t+c t^{2 /(1+\alpha)}\right)} \\
\kappa p=\frac{4 \alpha}{3(1+\alpha)^{2} t^{2}} .
\end{array}
$$

An equation of state means that $p$ uniquely depends on $\mu$. This takes place, if and only if $\alpha=0$ or $b / c=$ const. The latter is equivalent to the hypersurfacehomogeneity of the model and is of lower interest here. For $\alpha=0$ the initial model is the dust-filled EINSTEIN-DE SITTER model (EINSTEIN 1932). With (18) we obtain $p=0$ and $\mu \geq 0$ and may formulate:

If the EINSTEIN-DE SITTER universe is $x$-transformed into a perfect fluid model, then this model also contains dust and fulfils the energy conditions.

These models have the following form: inserting (17) with $\alpha=0$ into (6) we get a dust-filled model

$$
d s^{2}=-d t^{2}+t^{4 / 3}\left\{[b(x) / t+c(x)]^{2} d x^{2}+d y^{2}+d z^{2}\right\}
$$

with arbitrary b, $c$ as before. Equ. (19) is contained in SZEKERES (1975) as case (iii), but the parameter $\epsilon$ used there may be non-constant. As an illustration we give two examples of this model (19):

1. If $b=1, c>0$, then

$$
\kappa \mu=\frac{4 c}{3 t(1+c t)}
$$


hence the density contrast at two different values $x_{1}, x_{2}$ reads

$$
\frac{\mu_{1}}{\mu_{2}}=\frac{c_{2}}{c_{1}} \cdot \frac{1+c_{1} t}{1+c_{2} t}
$$

and tends to 1 as $t \rightarrow \infty$. This shows that one needs additional presumptions if one wants to prove an amplification of initial density fluctuations.

2. If $b=1, c=0$, then (19) is the KASNER vacuum solution. If now $c$ differs from zero in the neighbourhoods of two values $x_{1}, x_{2}$, then the model is built up from two thin dust slices and KASNER-like vacuum outside them. The invariant distance of the slices is

$$
A t^{-1 / 3}+B t^{2 / 3}
$$

with certain positive constants $A$ and $B$. This looks like gravitational repulsion, because the distance has a minimum at a positive value. But the $t^{-1 / 3}$-term is due to the partizipation of the slices in cosmological expansion and the remaining $t^{2 / 3}$-term is due to an attractive gravitational force in parabolic motion.

\section{Conclusion}

The transformation of a hypersurface-homogeneous cosmological model considered here firstly preserves all inner properties (expressed by the first fundamental form) of the slices $t=$ const., and secondly the property that $t$ is a synchronized time, but may change all other outer properties (essentially expressed by the second fundamental form). The investigations of sections 3. and 4. show that energy conditions are preserved under very special presumptions only.

One may consider these transformations as a guide in the search for new exact solutions of EINSTEIN's field equations. The new models are close to the initial hypersurface-homogeneous ones, if the transformation is close 
to the identical one. Thus, one can perturb a BIANCHI model with exact solutions without use of any (uncertain) approximations.

I want to thank Professor TREDER and Dr. v. BORZESZKOWSKI for helpful discussions.

\section{References}

EINSTEIN, A., W. DE SITTER: 1932, Proc. Nat. Acad. Sci. 18, 213.

SCHMIDT, H.-J.: 1982, Astron. Nachr. 303, 231.円

SZEKERES, P.: 1975, Commun. Math. Phys. 41, 55.

TOLMAN, R. C.: 1934, Relativity Thermodynamics and Cosmology, §164, Oxford.

WAINWRIGHT, J.: 1981, J. Phys. A 14, 1131.

(Received 1982 January 22)

In this reprint (done with the kind permission of the copyright owner) we removed only obvious misprints of the original, which was published in Astronomische Nachrichten: Astron. Nachr. 303 (1982) Nr. 5, pages 283 - 285; Author's address that time: Zentralinstitut für Astrophysik der AdW der DDR, 1502 PotsdamBabelsberg, R.-Luxemburg-Str. 17a.

\footnotetext{
${ }^{1}$ This is a misprint in the original, the cited paper is at page 227 and not at page 231; I thank Malcolm MacCallum for his note.
} 